\title{
Metacognición y Competencia investigativa en estudiantes del décimo ciclo, Escuela de Comunicación Lingüística y Literatura, Facultad de Educación, UNASAM - Huaraz, 2019
}

\author{
Metacognition and investigative competence in students of the tenth cycle, School of Linguistic \\ Communication and Literature, Faculty of Education, UNASAM - Huaraz, 2019
}

Violeta Mercedes Machaca Cano', Abelardo Rodolfo Campana Concha²

\begin{abstract}
RESUMEN
Objetivo: Describir la relación entre la metacognición y la competencia investigativa en estudiantes del décimo ciclo, Escuela de Comunicación Lingüística y Literatura, Facultad de Educación, UNASAM - Huaraz, 2019. Materiales y Método: Este estudio correlacional aplicó un cuestionarios para cada variable a una muestra de 80 estudiantes, luego procesada en el programa SPSS. Resultados: La hipótesis general halló una correlación de Rho $=0,904$ resultado encontrado por la opinión mayoritaria de los estudiantes, que en resumen entendemos que si la metacognición es aplicada casi siempre se alcanzará un adecuado desarrollo de las competencias investigativas. Las siete hipótesis específicas determinaron una relación de $\mathrm{Rho}=0,749, \mathrm{Rho}=0,727, \mathrm{Rho}=$ 0,434 , Rho $=0,588$, Rho $=0,617$, Rho $=0,499$ y Rho $=0,537$, respectivamente. Conclusión: La metacognición, aplicado casi siempre por la mayoría de la muestra; está relacionado con las competencias investigativas referido de adecuado. Con lo cual se concluye que la forma en la que la institución refuerza la metacognición de los estudiantes resulta suficiente para sus habilidades investigativas, sin embargo aún no alcanzan su capacidad máxima.
\end{abstract}

Palabras clave: Metacognición, competencias, investigación, cognitiva, observacional.

\section{ABSTRACT}

Objective: To describe the relationship between metacognition and research competence in students of the tenth cycle, School of Linguistic Communication and Literature, School of Education, UNASAM - Huaraz, 2019. Materials and Method: This correlational study applied a questionnaire for each variable to a sample of 80 students, and then processed in the SPSS program. Results: The general hypothesis found a correlation of $\mathrm{Rho}=0,904$, a result found by the majority opinion of the students, which in summary we understand that if metacognition is applied, an adequate development of research skills will almost always be achieved. The seven specific hypotheses determined a relationship of $\mathrm{Rho}=0,749$, $\mathrm{Rho}=0,727$, $\mathrm{Rho}=0,434$, $\mathrm{Rho}=0,588, \mathrm{Rho}=0,617, \mathrm{Rho}=0,499$ and Rho $=0,537$, respectively. Conclusion: Metacognition, applied usually by the majority of the sample; It is related to the investigative competences referred to as adequate. With which it is concluded that the way in which the institution reinforces the metacognition of the students is sufficient for their investigative abilities, however they still do not reach their maximum capacity.

Keywords: Metacognition, competences, research, cognitive, observational.

\section{INTRODUCCIÓN}

La Educación Superior, en las últimas décadas, sobre todo desde los años 90 del siglo pasado dejó que sus estudiantes investiguen, debido a que no era requisito para graduarse, dejando de lado la investigación que es uno de los aspectos muy importantes para lograr el desarrollo del país en todo los aspectos. UNESCO (2009), señala, la investigación científica, es en este sentido la mejor vía para producir conocimientos que permitan la consecución de tal fin pág. 2.

Por otro lado, tampoco el estado invierte en la investigación sobre todo, en los centros de formación universitaria, a pesar, el estatuto universitario señala, de acuerdo a la Ley el egresado debe poseer capacidades investigativas.

Según la Ley N³0220, la universidad tiene por objetivo:

"Formar profesionales de alta calidad de manera integral y con pleno sentido de responsabilidad social de acuerdo a las necesidades del país. Además de realizar y promover la investigación científica, tecnológica y humanística, la creación intelectual y artística".

Por otro lado, se observa que los estudiantes del nivel superior carecen en su mayoría de muchas competencias, así como valorar sus propios conocimientos, hasta qué punto logran aprender, a partir de ello puedan superar sus aprendizajes valorando y reconociendo mediante la metacognición sus saberes previos, y los adquiridos en las aulas universitarias, por lo que es necesario realizar cambios de paradigmas, con la finalidad de fortalecer las competencias investigativas de los futuros profesionales, quienes deberían aportar con creses en el desarrollo personal, de las carrearas profesionales, en este caso sobre todo en el campo educativo, donde los estudiantes estén bien capacitados e incorporarse al campo laboral con eficiencia, la solvencia de resolver problemas.

Estas variables fueron estudiadas y apoyadas de estudios anteriores como los de Rebaza (2016) quien en su investigación describió la relación entre las estrategias 
metacognitivas, aprendizaje autorregulado y autoestima en los estudiantes, por lo cual considera necesario que los docentes sepan enseñar en base de estrategias metacognitivas y puedan ellos producir sus propios conocimientos mediante la investigación, asimismo es necesario que los profesionales de la educación motiven a los estudiantes a producir nuevos conocimientos, ya que es un proceso y lo solo un acto inmediato.

Páez (2016) analizó las competencias investigativas en la educación superior y evidenció la importancia de fortalecerlas e implementar estrategias didácticas, sin embargo señala que a veces las clases resultan insuficientes para los estudiantes, para lo cual la institución necesita de cursos adicionales y docentes que sepan de investigación para profundizar distintos temas.

Por tanto, el objetivo de esta investigación fue Describir la relación entre la metacognición y la competencia investigativa en estudiantes universitarios.

\section{MÉTODO}

Corresponde a la investigación del enfoque cuantitativo, nivel explicativo, ex pos facto, que centra su análisis correlacional entre la variable metacognición y competencia investigativa. El tipo de investigación es no experimental con un diseño correlacional. La muestra estuvo conformada por 80 estudiantes del décimo ciclo de la Escuela de Comunicación Lingüística y Literatura, Facultad de Educación, UNASAM - Huaraz. Los datos obtenidos fueron procesadas en el programa SPSS.

\section{RESULTADOS}

\section{Tabla 1}

Frecuencias variable 1: metacognición

\begin{tabular}{llrr}
\hline & & Frecuencia & Porcentaje \\
\hline \multirow{4}{*}{ Válidos } & Casi nunca & 1 & 1,2 \\
& A veces & 35 & 43,8 \\
& Casi siempre & 44 & 55,0 \\
\cline { 2 - 4 } & Total & & \\
\hline
\end{tabular}

El $55,0 \%$ indican casi siempre se desarrolla la metacognición, $43,8 \%$ señalan a veces, $1,2 \%$ casi nunca, evidencia la mayoría no domina la variable mencionada.

\section{Prueba De Hipótesis General}

Hg1. La metacognición se relaciona positiva y significativamente con la competencia investigativa en los estudiantes del décimo semestre.

HGO. La metacognición no se relaciona positiva y significativamente con la competencia investigativa en los estudiantes del décimo semestre.

\section{Tabla 2}

Grado correlación entre metacognición y competencia investigativa

\begin{tabular}{|c|c|c|c|}
\hline \multicolumn{2}{|c|}{ Correlación } & \multicolumn{2}{|l|}{$\begin{array}{l}\text { Competencias } \\
\text { investigativas }\end{array}$} \\
\hline \multirow{3}{*}{$\begin{array}{l}\text { Rho de } \\
\text { Spearman }\end{array}$} & \multirow{3}{*}{ Metacognición } & $\begin{array}{l}\text { Coeficiente de } \\
\text { correlación }\end{array}$ & 0,904 \\
\hline & & Sig. (bilateral) & 0,000 \\
\hline & & $\mathrm{N}$ & 80 \\
\hline
\end{tabular}

Se identificó un nivel de correlación muy buena de Rho = 0,904 . entre las variables estudiadas con una tendencia de regular según la afirmación de la muestra.

\section{Hipótesis específica 1}

H1. La metacognición se relaciona significativamente con la dimensión cognitiva de la competencia investigativa de los estudiantes.

\section{Tabla 3}

Correlación entre metacognición y dimensión comunicativa

\begin{tabular}{|c|c|c|c|}
\hline \multicolumn{2}{|c|}{ Correlación } & \multicolumn{2}{|l|}{ Cognitiva } \\
\hline \multirow{3}{*}{$\begin{array}{l}\text { Rho de } \\
\text { Spearman }\end{array}$} & \multirow{3}{*}{ Metacognición } & $\begin{array}{l}\text { Coeficiente de } \\
\text { correlación }\end{array}$ & 0,749 \\
\hline & & Sig. (bilateral) & 0,000 \\
\hline & & $\mathrm{N}$ & 80 \\
\hline
\end{tabular}

Se identificó un nivel de relación buena de Rho $=0,7, p=$ 0,000 , permite aceptar la hipótesis alterna. De acuerdo a las respuestas vertidas manifiesta una tendencia de regular

\section{Hipótesis específica 2}

H2. La metacognición se relaciona significativamente con la dimensión observacional de la competencia investigativa de los estudiantes.

\section{Tabla 4}

Grado de Correlación entre metacognición y dimensión observacional

\begin{tabular}{|c|c|c|c|}
\hline \multicolumn{2}{|c|}{ Correlación } & \multicolumn{2}{|l|}{ Observacional } \\
\hline \multirow{3}{*}{$\begin{array}{l}\text { Rho de } \\
\text { Spearman }\end{array}$} & \multirow{3}{*}{ Metacognición } & $\begin{array}{l}\text { Coeficiente de } \\
\text { correlación }\end{array}$ & 0,727 \\
\hline & & Sig. (bilateral) & 0,000 \\
\hline & & $\mathrm{N}$ & 80 \\
\hline
\end{tabular}


Se identificó un nivel de relación buena de Rho $=0,727$ y el $p=0,000$, se acepta la hipótesis alterna.

\section{Hipótesis específica 3}

H3. La metacognición se relaciona significativamente con la dimensión analítica de la competencia investigativa de los estudiantes.

\section{Tabla 5}

Correlación entre metacognición y dimensión analítica.

\begin{tabular}{|c|c|c|c|}
\hline \multicolumn{2}{|c|}{ Correlación } & \multicolumn{2}{|l|}{ Analítica } \\
\hline \multirow{3}{*}{$\begin{array}{l}\text { Rho de } \\
\text { Spearman }\end{array}$} & \multirow{3}{*}{ Metacognición } & $\begin{array}{l}\text { Coeficiente de } \\
\text { correlación }\end{array}$ & 0,434 \\
\hline & & Sig. (bilateral) & 0,000 \\
\hline & & $\mathrm{N}$ & 80 \\
\hline
\end{tabular}

Se halló un nivel de relación moderada $\mathrm{Rho}=0,434, \mathrm{p}=$ 0,000 , aceptándose la hipótesis alterna.

Hipótesis específica 4

H4. La metacognición se relaciona significativamente con la dimensión procedimental de la competencia investigativa de los estudiantes.

\section{Tabla 6}

Nivel de relación entre la metacognición y procedimental

\begin{tabular}{|c|c|c|c|}
\hline \multicolumn{2}{|c|}{ Correlación } & \multicolumn{2}{|l|}{ Procedimental } \\
\hline \multirow{3}{*}{$\begin{array}{l}\text { Rho de } \\
\text { Spearman }\end{array}$} & \multirow{3}{*}{ Metacognición } & $\begin{array}{l}\text { Coeficiente de } \\
\text { correlación }\end{array}$ & 0,588 \\
\hline & & Sig. (bilateral) & 0,000 \\
\hline & & $\mathrm{N}$ & 80 \\
\hline
\end{tabular}

Se identificó un nivel de relación moderada de Rho = $0,588, p=0,000$, se afirma la hipótesis alterna.

\section{Hipótesis específica 5}

H5. La metacognición se relaciona significativamente con la dimensión comunicativa de la competencia investigativa de los estudiantes.

\section{Tabla 7}

Correlación entre metacognición y dimensión comunicativa

\begin{tabular}{|c|c|c|c|}
\hline \multicolumn{2}{|c|}{ Correlación } & \multicolumn{2}{|l|}{ Comunicativa } \\
\hline \multirow{3}{*}{$\begin{array}{l}\text { Rho de } \\
\text { Spearman }\end{array}$} & \multirow{3}{*}{ Metacognición } & $\begin{array}{l}\text { Coeficiente de } \\
\text { correlación }\end{array}$ & 0,617 \\
\hline & & Sig. (bilateral) & 0,000 \\
\hline & & $\mathrm{N}$ & 80 \\
\hline
\end{tabular}

Se identificó un nivel de relación buena de $\mathrm{Rho}=0,617, \mathrm{p}$ $=0,000$, permitiendo aceptar la hipótesis alterna.

\section{Hipótesis específica 6}

H6. La metacognición se relaciona significativamente con la dimensión tecnológica de la competencia investigativa de los estudiantes.

\section{Tabla 8}

Relación entre metacognición y la dimensión tecnológica

\begin{tabular}{|c|c|c|c|}
\hline \multicolumn{2}{|c|}{ Correlación } & \multicolumn{2}{|l|}{ Tecnológica } \\
\hline \multirow{3}{*}{$\begin{array}{l}\text { Rho de } \\
\text { Spearman }\end{array}$} & \multirow{3}{*}{ Metacognición } & $\begin{array}{l}\text { Coeficiente de } \\
\text { correlación }\end{array}$ & 0,499 \\
\hline & & Sig. (bilateral) & 0,000 \\
\hline & & $\mathrm{N}$ & 80 \\
\hline
\end{tabular}

Se identificó un nivel de relación moderada de Rho = 0,499 , la significancia $p=0,000$ afirmándose la hipótesis alterna, por tanto la metacognición tiene una frecuencia de casi siempre relacionándose como adecuado respecto a la competencia tecnológica.

\section{Hipótesis específica 7}

H7. La metacognición se relaciona significativamente con la dimensión interpersonal de la competencia investigativa de los estudiantes.

\section{Tabla 9}

Correlación entre la metacognición y la dimensión interpersonal

\begin{tabular}{|c|c|c|c|}
\hline \multicolumn{2}{|c|}{ Correlación } & \multicolumn{2}{|l|}{ Interpersonal } \\
\hline \multirow{3}{*}{$\begin{array}{l}\text { Rho de } \\
\text { Spearman }\end{array}$} & \multirow{3}{*}{ Metacognición } & $\begin{array}{l}\text { Coeficiente de } \\
\text { correlación }\end{array}$ & 0,537 \\
\hline & & Sig. (bilateral) & 0,000 \\
\hline & & $\mathrm{N}$ & 80 \\
\hline
\end{tabular}

Se identificó un nivel de relación moderada de Rho = 0,537 y el $p=0,000$, permite rechazar la hipótesis nula y aceptar la hipótesis alterna.

\section{DISCUSIÓN}

Al analizar las variables, hipótesis general halló una relación muy buena de Rho $=0,904$, explica la metacognición, 90,4\% realiza a veces la competencias investigativas.

Hipótesis específica segunda, se halló Rho $=0,727$; $72,7 \%$ opinan como a veces la dimensión observacional. Hipótesis específica tercera, se halló Rho $=0,434$, dimensión analítica 42,4\% considera adecuado. Cuarta hipótesis específica, se encontró Rho $=0,588$, la 
dimensión procedimental $59 \%$ considera regular.

Hipótesis específica quinta se determinó Rho = 0,617. En cuanto la dimensión comunicativa 60 \% opina en forma regular.

Hipótesis específica sexta se determinó Rho $=0,499$, respecto la dimensión tecnológica el 50,0\%. Menciona regular. Hipótesis específica séptima se determinó Rho = 0,537, referente la dimensión interpersonal el 54\% considera adecuada.

Analizando estos resultados de Díaz (2014) señala que la metacognición de desarrolla con el apoyo docente, ya que necesita su guía para su adecuado proceso, en este caso coincidimos en los resultados, ya que también evidenciamos que no siempre el docente está apoyando al estudiante a generar sus propios puntos de vista, alertando la necesidad de mayor compromiso docente para que el estudiante manifieste sus propios conocimientos en clase.

Con respecto a las competencias investigativas tenemos a Xiao (2018) quien considera que las estrategias de enseñanza cumplen un rol importante en las competencias investigativas en la educación superior, sin embargo al brindarse de forma regular perjudica el fortalecimiento de dichas competencias, adicionando nuestros resultados es necesario un compromiso de la institución capacitar al personal docente en temas investigación o en todo caso seleccionar idóneamente para que los estudiantes tengan los elementos altamente adecuados para sus formación.

\section{CONCLUSIONES}

1. La forma de reforzar institución en cuanto la metacognición en estudiantes resulta insuficiente para lograr habilidades investigativas.

2. Asimismo, resulta insuficiente desarrollar la capacidad de la observación, descripción, fuentes y procesamiento de la información sobre un tema específico.

3. En cuanto la capacidad para explicar sus observaciones, distingue los aspectos más importantes de un tema.

4. En el desarrollo de su capacidad para realizar análisis investigativos profundos, así como el establecimiento de categorías, selección de metodología, identificación del tipo de investigación.

5. La institución incentiva poco la generación conocimientos, resultando insuficiente para diseñar, comprobar y sistematizar una investigación.

6. En la generación de sus propios razonamientos resulta relativamente insuficiente como: redactar, brindar aportes, resumir en un artículo una investigación científica.

7. La generación de sus propios conocimientos resulta relativamente insuficiente para seleccionar la técnica para recolectar datos, usar softwares estadísticos, interpretar datos estadísticos y organizar sus resultados investigativos.

8. La capacidad para saber identificar el adecuado manejo de las fuentes y colaborar dentro de un trabajo de investigación.

\section{REFERENCIAS BIBLIOGRAFICAS}

Díaz, E. (2014). Implementación de estrategias metacognitivas para un aprendizaje significativo (Tesis Posgrado). Tecnológico de Monterrey, México. $\mathrm{R}$ e c u p e r a d o d e https://repositorio.tec.mx/bitstream/handle/11285/62249 2/02Ernesto\%20D\%C3\%ADaz\%20Esteves.pdf?sequen ce=1\&isAllowed=y

Diario Oficial El Peruano, Ley Nº 30220.Lima, Perú, 9 de julio de 2014.

Páez, J. (2016). Desarrollo de competencias investigativas basadas en la concepción sistémica de ambiente, en estudiantes de la escuela Normal Superior de Montería (Tesis Posgrado). Universidad Nacional de Colombia, Medellín. Recuperado de http://www.bdigital.unal.edu.co/55895/1/desarrollocomp etenciasinvestigativas.pdf

Rebaza, E. (2016). Relación entre estrategias metacognitivas, aprendizaje autorregulado y autoestima en los estudiantes en el Instituto Superior Pedagógico Indoamérica 2011 (Tesis Posgrado) Universidad Privada Antenor Orrego, Trujillo, Perú.

Unesco (2009). La integración de las tecnologías de la información y comunicación en los sistemas educativos. Buenos Aires: Unesco.

Xiao, J. (2018). Estrategias de enseñanza y competencias investigativas de los estudiantes de pos grado - Facultad de Educación - UNMSM, 2017 (Tesis Posgrado). UNMSM, Lima, Perú. 